\title{
On Waring's problem with polynomial summands II
}

by

\section{Hong Bing Yu (Hefei)}

1. Introduction. Let $f_{k}(x)$ be an integral-valued polynomial of degree $k$ with positive leading coefficient, $f_{k}(0)=0$ and satisfying the condition that there do not exist integers $c$ and $q>1$ such that $f_{k}(x) \equiv c(\bmod q)$ identically. It is known that $f_{k}(x)$ is of the form

$$
f_{k}(x)=a_{k} F_{k}(x)+\ldots+a_{1} F_{1}(x),
$$

where $F_{i}(x)=x(x-1) \ldots(x-i+1) / i !(1 \leq i \leq k)$, and $a_{1}, \ldots, a_{k}$ are integers satisfying

$$
\left(a_{1}, \ldots, a_{k}\right)=1 \quad \text { and } \quad a_{k}>0 .
$$

Let $G\left(f_{k}\right)$ be the least $s$ such that the equation

$$
f_{k}\left(x_{1}\right)+\ldots+f_{k}\left(x_{s}\right)=n, \quad x_{i} \geq 0,
$$

is soluble for all sufficiently large integers $n$. The problem of estimation for $G\left(f_{k}\right)$ has been investigated by many authors (see Wooley [6] for references). Here we remark only that Hua [3] has shown that $G\left(f_{k}\right) \leq(k-1) 2^{k+1}$; and, if

$$
H_{k}(x)=2^{k-1} F_{k}(x)-2^{k-2} F_{k-1}(x)+\ldots+(-1)^{k-1} F_{1}(x), \quad k \geq 4,
$$

then $G\left(H_{k}\right)=2^{k}-\frac{1}{2}\left(1-(-1)^{k}\right)$. In [3] Hua conjectured further that generally

$$
G\left(f_{k}\right) \leq 2^{k}-\frac{1}{2}\left(1-(-1)^{k}\right) .
$$

This was confirmed in [7] for $k=4,5$ and 6 . The purpose of this paper is to prove that (1.5) is true for all $k \geq 7$. In fact, we prove the following slightly more precise result.

Theorem 1. Let $H_{k}(x)$ be as in (1.4). For $k \geq 6$, if $f_{k}(x)$ satisfies

$$
2 \nmid f_{k}(1) \quad \text { and } \quad f_{k}(x) \equiv(-1)^{k-1} f_{k}(1) H_{k}(x)\left(\bmod 2^{k}\right) \quad \text { for any } x,
$$

1991 Mathematics Subject Classification: Primary 11P05.

Project supported by the National Natural Science Foundation of China. 
then $G\left(f_{k}\right)=2^{k}-1$ for odd $k$ and $2^{k}-1$ or $2^{k}$ for even $k$; otherwise,

$$
G\left(f_{k}\right) \leq 2^{k-1}+4(k-1) .
$$

In order to investigate the solubility of (1.3), we define $\mathfrak{S}^{*}\left(f_{k}\right)$ to be the least number such that if $s \geq \mathfrak{S}^{*}\left(f_{k}\right)$ then $\mathfrak{S}_{s}\left(f_{k}, n\right) \geq c$ for some positive $c$ independent of $n$, where $\mathfrak{S}_{s}\left(f_{k}, n\right)$ is the singular series corresponding to the equation (1.3) (see Hua [2] and the remark of Wooley [6]). We also define $G^{*}\left(f_{k}\right)$ to be the least number $s$ with the property that all sufficiently large numbers $n$ with $\mathfrak{S}_{s}\left(f_{k}, n\right) \geq c$ are represented in the form (1.3). From earlier works on $G^{*}\left(f_{k}\right)$ (see Hua [4]) we have, in particular,

$$
G^{*}\left(f_{k}\right)<2^{k-1}+4(k-1) \quad \text { for } k \geq 6 .
$$

(We remark that very sharp estimates on $G^{*}\left(f_{k}\right)$ for large $k$ have recently been obtained by Wooley [6].) Therefore, in view of (1.7) and (2.9) below, to prove Theorem 1 it suffices to prove the following result.

Theorem 2. For $k \geq 6$, if $f_{k}(x)$ satisfies (1.6), then $\mathfrak{S}^{*}\left(f_{k}\right) \leq 2^{k}-$ $\frac{1}{2}\left(1-(-1)^{k}\right)$; otherwise, $\mathfrak{S}^{*}\left(f_{k}\right) \leq 2^{k-1}+4(k-1)$.

We note that, for quartic and quintic polynomials, more precise results on $\mathfrak{S}^{*}\left(f_{k}\right)$ have been established in [7] and [8]:

If $f_{k}(x)(k=4$ and 5$)$ does not satisfy (1.6), then

$$
\max _{f_{4}} \mathfrak{S}^{*}\left(f_{4}\right)=11 \text { and } \max _{f_{5}} \mathfrak{S}^{*}\left(f_{5}\right)=16 .
$$

2. Notation and preliminary results. Let $f_{k}(x)$ be as in (1.1), and let $d$ be the least common denominator of the coefficients of $f_{k}(x)$. For each prime $p$, we define $t=t\left(f_{k}, p\right)$ by $p^{t} \| d$. Let $\theta=\theta\left(f_{k}, p\right)$ be the greatest integer such that

$$
p^{t} f_{k}^{\prime}(x) \equiv 0\left(\bmod p^{\theta}\right) \quad \text { for any } x
$$

and let $f_{k}^{*}(x)=p^{-\theta}\left(p^{t} f_{k}^{\prime}(x)\right)$. Define the integer $\delta=\delta(p, k)$ by

$$
p^{\delta} \leq k-1<p^{\delta+1},
$$

and let

$$
\gamma=\gamma\left(f_{k}, p\right)= \begin{cases}\theta-t+\delta+2 & \text { for } p=2, \\ \theta-t+\delta+1 & \text { for } p>2 .\end{cases}
$$

We record for later use that (see Hua [3, Lemma 3.3])

$$
\gamma \leq k+\delta+1 \text { for } p=2 \text { and } \gamma \leq\left[\frac{k}{p-1}\right]+\delta+1 \text { for } p \geq 3 .
$$

Let $M_{s}\left(f_{k}, p^{l}, n\right)$ denote the number of solutions of the congruence

$$
f_{k}\left(x_{1}\right)+\ldots+f_{k}\left(x_{s}\right) \equiv n\left(\bmod p^{l}\right), \quad 0 \leq x_{i}<p^{l+t},
$$


and let $\Gamma\left(f_{k}, p^{l}\right)$ be the least value of $s$ for which (2.5) is soluble for every $n$. From Hua [2, Section 7] we see that, if $s \geq 2 k+1$, to establish $\mathfrak{S}^{*}\left(f_{k}\right) \leq s$ it suffices to show that for all primes $p$ and any integers $n$ and $l \geq c$,

$$
M_{s}\left(f_{k}, p^{l}, n\right) \geq p^{(s-1)(l-c)},
$$

where $c$ is a positive constant depending only on $f_{k}(x)$. Since a direct treatment of (2.6) presents certain technical difficulties, we define $N_{s}\left(f_{k}, p^{l}, n\right)$ to be the number of solutions of the congruence (2.5) with the $f_{k}^{*}\left(x_{i}\right)$ not all divisible by $p$. Then (see [2, Lemma 7.6])

$$
N_{s}\left(f_{k}, p^{l}, n\right)=p^{(s-1)(l-\gamma)} N_{s}\left(f_{k}, p^{\gamma}, n\right) \quad \text { for } l \geq \gamma .
$$

Let $\Gamma^{*}\left(f_{k}, p^{\gamma}, n\right)$ be the least $s$ such that $N_{s}\left(f_{k}, p^{\gamma}, n\right) \geq 1$. Then, by (2.7) and $M_{s}\left(f_{k}, p^{l}, n\right) \geq N_{s}\left(f_{k}, p^{l}, n\right)$, (2.6) holds (with $c=\gamma$ ) when $s=\Gamma^{*}\left(f_{k}, p^{\gamma}, n\right)$. Moreover, we define $\Gamma^{*}\left(f_{k}, p^{\gamma}\right)=\max _{n} \Gamma^{*}\left(f_{k}, p^{\gamma}, n\right)$. Then, in particular, when $s=\Gamma^{*}\left(f_{k}, p^{\gamma}\right)$ the congruence (2.5) is soluble for any $n$ and $l \geq 1$. Also, by the definition, we have

$$
\Gamma\left(f_{k}, p^{\gamma}\right) \leq \Gamma^{*}\left(f_{k}, p^{\gamma}\right) \leq \Gamma\left(f_{k}, p^{\gamma}\right)+1 .
$$

Now we see that to prove Theorem 2 , it suffices to establish the following two results.

Theorem 3. Suppose $k \geq 6$.

(i) If $f_{k}(x)$ satisfies $(1.6)$, then

$$
\Gamma\left(f_{k}, 2^{k}\right)=2^{k}-1 ;
$$

and, when $s=2^{k}-\frac{1}{2}\left(1-(-1)^{k}\right)$, we have

$$
M_{s}\left(f_{k}, 2^{l}, n\right) \geq 2^{(s-1)(l-2 k)} \quad \text { for all } n \text { and } l \geq 2 k \text {. }
$$

(ii) Otherwise, we have $\Gamma^{*}\left(f_{k}, 2^{\gamma}\right) \leq 2^{k-1}+4(k-1)$.

TheOREM 4 . For $k \geq 6$ and prime $p \geq 3$, we have

$$
\Gamma^{*}\left(f_{k}, p^{\gamma}\right) \leq 2^{k-1}+4(k-1) .
$$

Our proof of Theorems 3 and 4 is motivated by Hua [3] and $\mathrm{Yu}$ [7] (see Sections 3 to 5 of this paper). Before proceeding further we record two lemmas. Lemma 2.1 (below) may be compared with Hua [3, Lemmas 4.4 and 4.5]. It follows from (1.1) and a simple calculation. Lemma 2.2 can be seen from the proof of Hua [3, Lemma 3.2] (see also Lovász [5, Problem 1.43(e)]).

Lemma 2.1. Let $f_{k}(x)$ be as in (1.1). Then

(i) $f_{k}(x+2)-f_{k}(x)=2 a_{k} F_{k-1}(x)+\sum_{i=1}^{k-1}\left(2 a_{i}+a_{i+1}\right) F_{i-1}(x)$ with $F_{0}(x)$ being interpreted as 1.

(ii) $f_{k}(x+1)+f_{k}(x)-f_{k}(1)=2 a_{k} F_{k}(x)+\sum_{i=1}^{k-1}\left(2 a_{i}+a_{i+1}\right) F_{i}(x)$. 
LEMma 2.2. Let

$$
P_{m}(x)=\sum_{i=1}^{m} \alpha_{i} F_{i}(x)
$$

and write $P_{m}^{\prime}(x)=\sum_{i=0}^{m-1} \beta_{i} F_{i}(x)$. Then $\beta_{i}(0 \leq i \leq m-1)$ are given by

$$
\beta_{i}=(-1)^{m-i-1}\left(\frac{\alpha_{m}}{m-i}-\frac{\alpha_{m-1}}{m-i+1}+\ldots+(-1)^{m-i-1} \alpha_{i+1}\right) .
$$

3. Proof of Theorem 3(i). In this section, we will use the notation introduced in Section 2 for $p=2$ only. Moreover, for an integral-valued polynomial $Q(x)$, we will define (for $p=2) t(Q), \theta(Q), \gamma(Q)$ and $Q^{*}(x)$ in the same way as $t=t\left(f_{k}, 2\right), \theta=\theta\left(f_{k}, 2\right), \gamma=\gamma\left(f_{k}, 2\right)$ and $f_{k}^{*}(x)$ for $f_{k}(x)$ in Section 2.

Suppose that $f_{k}(x)$ satisfies (1.6). Without loss of generality we may assume that $a_{1}=f_{k}(1)=(-1)^{k-1}$. Then, by (1.1) and (1.6),

$$
a_{i} \equiv(-1)^{k-i} 2^{i-1}\left(\bmod 2^{k}\right) \quad(2 \leq i \leq k) .
$$

It follows that

$$
2^{k} \| 2 a_{k} \quad \text { and } \quad 2^{k} \mid\left(2 a_{i}+a_{i+1}\right) \quad(1 \leq i \leq k-1) .
$$

By Lemma 2.1(i) and (3.2), we have

$$
f_{k}(x+2)-f_{k}(x) \equiv 0\left(\bmod 2^{k}\right) \quad \text { for any } x .
$$

Thus $f_{k}(x)$ takes only two different values, 0 and $(-1)^{k-1}$, mod $2^{k}$, and then (2.9) follows.

Let

$$
G_{k}(x)=2^{-k}\left(f_{k}(x+1)+f_{k}(x)-(-1)^{k-1}\right)
$$

and write

$$
G_{k}(x)=\sum_{i=1}^{k} b_{i} F_{i}(x) .
$$

By Lemma 2.1(ii) and (3.2), $b_{i}(1 \leq i \leq k)$ are integers and $2 \nmid b_{k}$.

Define integers $\tau$ and $\sigma$ by $2^{\tau} \| k$ ! and $2^{\sigma} \leq k<2^{\sigma+1}$. Since $2 \nmid b_{k}$, we have $t\left(G_{k}\right)=\tau$, and hence $\theta\left(G_{k}\right)=\tau-\sigma$ by Lemma 2.2. Thus $G_{k}^{*}(x)=2^{\sigma} G_{k}^{\prime}(x)$, and so

$$
G_{k}^{*}(x)=2^{-(k-\sigma)}\left(f_{k}^{\prime}(x+1)+f_{k}^{\prime}(x)\right)
$$

by (3.4). Furthermore, writing

$$
G_{k}^{*}(x)=\sum_{i=0}^{k-1} c_{i} F_{i}(x)
$$


with 2-adic integral $c_{i}(0 \leq i \leq k-1)$, we see from Lemma 2.2 that

$$
c_{i} \equiv \begin{cases}0(\bmod 2) & \text { for } i>k-2^{\sigma}, \\ b_{i+2^{\sigma}}(\bmod 2) & \text { for } 0 \leq i \leq k-2^{\sigma} .\end{cases}
$$

The following result is an analogue of Hua [3, Theorem 4].

Lemma 3.1. (i) The congruence

$$
G_{k}(x) \equiv A\left(\bmod 2^{l}\right), \quad 2 \nmid G_{k}^{*}(x),
$$

is soluble for any $A$ and $l \geq 1$.

(ii) If $2 \nmid G_{k}^{*}\left(x_{0}\right)$ for some $x_{0}$, then either $2 \nmid f_{k}^{*}\left(x_{0}\right)$ or $2 \nmid f_{k}^{*}\left(x_{0}+1\right)$.

Proof. We prove that, for any integers $x, y$ and $m \geq 0$,

$$
G_{k}\left(x+2^{m+\sigma} y\right)-G_{k}(x) \equiv 2^{m} y G_{k}^{*}(x)\left(\bmod 2^{m+1}\right)
$$

and

$$
G_{k}^{*}\left(x+2^{m+\sigma} y\right) \equiv G_{k}^{*}(x)\left(\bmod 2^{m+1}\right) .
$$

This suffices to prove part (i) by induction on $l$ (when $l=1$ the result follows immediately from (3.9) and (3.10) with $m=0)$.

We now prove (3.9). By Vandermonde's identity (see Lovász [5, Problem 1.45]), we have for $1 \leq i \leq k$,

$$
F_{i}\left(x+2^{m+\sigma} y\right)-F_{i}(x)=\sum_{j=1}^{i}\left(\begin{array}{c}
2^{m+\sigma} y \\
j
\end{array}\right) F_{i-j}(x) .
$$

It is easily seen that, for any integer $y$,

$$
\left(\begin{array}{c}
2^{m+\sigma} y \\
2^{\sigma}
\end{array}\right) \equiv 2^{m} y\left(\bmod 2^{m+1}\right)
$$

and

$$
\left(\begin{array}{c}
2^{m+\sigma} y \\
j
\end{array}\right) \equiv 0\left(\bmod 2^{m+1}\right) \quad \text { for } j \neq 2^{\sigma}
$$

(note $j \leq k<2^{\sigma+1}$ ). Hence

$$
F_{i}\left(x+2^{m+\sigma} y\right)-F_{i}(x) \equiv 2^{m} y F_{i-2^{\sigma}}(x)\left(\bmod 2^{m+1}\right)
$$

for any integers $x$ and $y$ (where $F_{j}(x)$ with $j<0$ is interpreted to be 0 ). From this, (3.5), (3.7) and (3.8) we have

$$
\begin{aligned}
G_{k}\left(x+2^{m+\sigma} y\right)-G_{k}(x) & \equiv \sum_{i=1}^{k} 2^{m} y b_{i} F_{i-2^{\sigma}}(x) \equiv \sum_{i=0}^{k-2^{\sigma}} 2^{m} y b_{i+2^{\sigma}} F_{i}(x) \\
& \equiv \sum_{i=0}^{k-2^{\sigma}} 2^{m} y c_{i} F_{i}(x) \equiv 2^{m} y G_{k}^{*}(x)\left(\bmod 2^{m+1}\right),
\end{aligned}
$$

as required. (3.10) can be proved similarly. 
To prove (ii), we note that now $t=0$, so (3.6) implies that $\theta \leq k-\sigma$. If $\theta=k-\sigma$, then $G_{k}^{*}(x)=f_{k}^{*}(x+1)+f_{k}^{*}(x)$, and the result follows at once. Suppose that $\theta \leq k-\sigma-1$. By (3.2), Lemmas 2.1(i) and 2.2 we have

$$
f_{k}^{\prime}(x+2)-f_{k}^{\prime}(x) \equiv 0\left(\bmod 2^{k-\delta}\right) \quad \text { for any } x .
$$

(Recall that in this section $\delta$ satisfies $2^{\delta} \leq k-1<2^{\delta+1}$.) Clearly $\delta \leq \sigma$, so that $2^{\theta+1} \mid 2^{k-\delta}$. It follows from (3.11) that $2 \nmid f_{k}^{*}(x)$ either for all odd $x$ or for all even $x$, and therefore the desired result also follows.

Our next step is to establish the results analogous to Hua [3, Lemmas 4.6-4.8]. We define

$$
E_{k}(x)=2^{-k} f_{k}(2 x) \quad \text { and } \quad O_{k}(x)=2^{-k}\left(f_{k}(2 x+1)-(-1)^{k-1}\right) .
$$

By (3.3), both $E_{k}(x)$ and $O_{k}(x)$ are integral-valued polynomials. We write

$$
E_{k}(x)=\sum_{i=1}^{k} d_{i} F_{i}(x) \quad \text { and } \quad O_{k}(x)=\sum_{i=1}^{k} d_{i}^{\prime} F_{i}(x) .
$$

Lemma 3.2. (i) If $k \geq 7$ is odd, then neither $E_{k}(x)$ nor $O_{k}(x)$ is constant modulo 2 , and $\gamma\left(E_{k}\right) \leq(k-1) / 2+\delta$ and $\gamma\left(O_{k}\right) \leq(k-1) / 2+\delta$.

(ii) If $k \geq 8$ is even, then either $E_{k}(x)$ is not constant modulo 2 and $\gamma\left(E_{k}\right) \leq k / 2+\delta$ or $O_{k}(x)$ is not constant modulo 2 and $\gamma\left(O_{k}\right) \leq k / 2+\delta$.

Pr o of. From Kemmer's identity (see Gupta [1, Chapter 8, §9.2]) it follows that

$$
F_{l}(2 x)=\sum_{i \leq l} 2^{2 i-l}\left(\begin{array}{c}
i \\
l-i
\end{array}\right) F_{i}(x) \quad \text { for any } x .
$$

Then by (1.1) we have

$$
f_{k}(2 x)=\sum_{i=1}^{k} F_{i}(x) \sum_{l=i}^{\min (2 i, k)} a_{l} 2^{2 i-l}\left(\begin{array}{c}
i \\
l-i
\end{array}\right) .
$$

This, together with $F_{l}(2 x+1)=F_{l}(2 x)+F_{l-1}(2 x)$, gives

$$
\begin{aligned}
f_{k}(2 x+1)- & (-1)^{k-1} \\
& =f_{k}(2 x)+\sum_{i=1}^{k-1} F_{i}(x) \sum_{l=i}^{\min (2 i, k-1)} a_{l+1} 2^{2 i-l}\left(\begin{array}{c}
i \\
l-i
\end{array}\right) .
\end{aligned}
$$

Now by (3.1) and (3.12) to (3.15) we see that

$$
2^{k-1} \mid\left(d_{k}, d_{k}^{\prime}\right) \quad \text { and } \quad 2^{k-3} \mid\left(d_{k-1}, d_{k-1}^{\prime}\right) .
$$

Also, we have $2 \nmid d_{(k+1) / 2}$ and $2 \nmid d_{(k+1) / 2}^{\prime}$ for odd $k$, thus the first assertion of (i) follows. Further, by $2 \nmid d_{(k+1) / 2},(3.16)$ and Lemma 2.2 , it can be proved easily that $\theta\left(E_{k}\right) \leq k-(k+1) / 2-2+t\left(E_{k}\right)$ for $k \geq 7$ (cf. the proof of Hua 
[3, Lemma 3.2]). Thus $\gamma\left(E_{k}\right) \leq(k-1) / 2+\delta$ (cf. (2.3)). The same argument gives $\gamma\left(O_{k}\right) \leq(k-1) / 2+\delta$.

If $k$ is even, then either $2 \nmid d_{k / 2}$ or $2 \nmid d_{k / 2}^{\prime}$. The assertions of (ii) follow as above.

We are now in a position to prove the second assertion of Theorem 3(i).

(I) $k$ is odd. Let $s=2^{k}-1$, and for any $n$ let $r_{n}$ be the integer satisfying $n \equiv r_{n}\left(\bmod 2^{k}\right)$ and $0 \leq r_{n}<2^{k}$. We consider several cases.

(i) $1 \leq r_{n} \leq 2^{k}-2$. By Lemma 3.1(i) the congruence

$$
G_{k}(x)+\sum_{i=2}^{r_{n}} O_{k}\left(y_{i}\right) \equiv m\left(\bmod 2^{l}\right), \quad 2 \nmid G_{k}^{*}(x),
$$

is soluble for any $m, y_{i}\left(2 \leq i \leq r_{n}\right)$ and $l \geq 1$. Hence in case (i) we have, by (3.4), (3.12) and Lemma 3.1(ii),

$$
\Gamma^{*}\left(f_{k}, 2^{\gamma}, n\right) \leq r_{n}+1 \leq 2^{k}-1,
$$

which implies that $N_{s}\left(f_{k}, 2^{\gamma}, n\right) \geq 1$, and the result follows immediately (cf. Section 2 and note that $\gamma<2 k$ by $(2.4)$ for $p=2$ ).

(ii) $r_{n}=0$. We note that, by Lemma $3.2(\mathrm{i}), s>2^{\gamma\left(E_{k}\right)}$ for $k \geq 7$. Thus, by the Davenport-Chowla lemma (cf. [7, Lemma 2.2]), for $l=\gamma\left(E_{k}\right)$ the congruence

$$
\sum_{i=1}^{s} E_{k}\left(x_{i}\right) \equiv m\left(\bmod 2^{l}\right)
$$

has a solution with $2 \nmid E_{k}^{*}\left(x_{1}\right)$, i.e. $N_{s}\left(E_{k}, 2^{\gamma\left(E_{k}\right)}, m\right) \geq 1$, for any $m$. Thus the number $M_{s}\left(E_{k}, 2^{l}, m\right)$ of solutions of the congruence (3.17) is at least $2^{(s-1)\left(l-\gamma\left(E_{k}\right)\right)}$ for all $m$ and $l \geq k>\gamma\left(E_{k}\right)$ (cf. Section 2). Hence, in view of (3.12), the result holds in case (ii).

(iii) $r_{n}=2^{k}-1$. The same argument as in (ii) with $E_{k}(x)$ replaced by $O_{k}(x)$ shows that $N_{s}\left(O_{k}, 2^{\gamma\left(O_{k}\right)}, m\right) \geq 1$ for all $m$, and the result also follows in case (iii).

(II) $k$ is even. When $k=6$ the result has been proved in [7]. For $k \geq 8$ let $s=2^{k}$, and for any $n$ let $r_{n}$ be the integer satisfying $n \equiv-r_{n}\left(\bmod 2^{k}\right)$ and $0 \leq r_{n}<2^{k}$.

When $1 \leq r_{n} \leq 2^{k}-1$, in a similar way to (I)(i), we have $\Gamma^{*}\left(f_{k}, 2^{\gamma}, n\right) \leq$ $r_{n}+1 \leq 2^{k}$ and hence the result. Moreover, by Lemma 3.2(ii) and a similar argument to (I)(ii), it is easily seen that either $N_{s}\left(E_{k}, 2^{\gamma\left(E_{k}\right)}, m\right) \geq 1$ or $N_{s}\left(O_{k}, 2^{\gamma\left(O_{k}\right)}, m\right) \geq 1$, for all $m$. Thus for $r_{n}=0$ the desired result also holds.

The proof of Theorem 3(i) is now complete. 
4. Proof of Theorem 3(ii). We need the following simple lemma.

LEMma 4.1. Let $\lambda$ be the greatest integer such that

$$
f_{k}(x+2)-f_{k}(x) \equiv 0\left(\bmod 2^{\lambda}\right) \quad \text { for any } x .
$$

Then $\lambda \leq k$, and equality holds if and only if $f_{k}(x)$ satisfies (1.6).

Proof. By Lemma 2.1(i), we have

(4.1) $2 a_{k} \equiv 0\left(\bmod 2^{\lambda}\right)$ and $2 a_{i}+a_{i+1} \equiv 0\left(\bmod 2^{\lambda}\right) \quad(1 \leq i \leq k-1)$.

Then by contradiction and (1.2) it follows that $\lambda \leq k$. Further, if $\lambda=k$, then it is easily seen by (4.1) and induction on $i$ that $a_{i} \equiv(-2)^{i-1} a_{1}\left(\bmod 2^{k}\right)$ for $2 \leq i \leq k$. Hence (1.6) follows. The converse result has already been proved in Section 3 (cf. (3.3)).

We now prove Theorem 3(ii) by induction. We note that by $\mathrm{Yu}[7$, Section 5 ] both (i) and (ii) of Theorem 3 hold for $k=5$. Suppose that $k \geq 6$ and that Theorem 3(ii) is true for polynomials of degree $k-1$. We then prove

$$
\Gamma\left(f_{k}, 2^{\gamma}\right) \leq 2^{k-1}+4(k-1)-1
$$

for any $f_{k}(x)$ not satisfying (1.6), which, in view of (2.8), completes our proof.

Since $f_{k}(x)$ does not satisfy (1.6), we have $\lambda \leq k-1$ by Lemma 4.1. If $\gamma \leq \lambda$ the result is trivial. Thus we may assume that $\gamma>\lambda$. By the definition of $\lambda$, there exists an integer $x_{0}$ such that $f_{k}\left(x_{0}+2\right)-f_{k}\left(x_{0}\right) \not \equiv 0$ $\left(\bmod 2^{\lambda+1}\right)$. By the Davenport-Chowla lemma we see that, when $l=2^{\lambda}-1$, the congruence

$$
f_{k}\left(x_{1}\right)+\ldots+f_{k}\left(x_{l}\right) \equiv n-m f_{k}\left(x_{0}\right)\left(\bmod 2^{\lambda}\right)
$$

is soluble for any $m$ and $n$.

The next step is to consider the solubility of the congruence

$$
f_{k}\left(x_{0}+2 y_{1}\right)+\ldots+f_{k}\left(x_{0}+2 y_{m}\right) \equiv m f_{k}\left(x_{0}\right)+2^{\lambda} A\left(\bmod 2^{\gamma}\right)
$$

for any $A$. We write

$$
g_{k}(y)=2^{-\lambda}\left(f_{k}\left(x_{0}+2 y\right)-f_{k}\left(x_{0}\right)\right) ;
$$

then (4.4) is equivalent to

$$
g_{k}\left(y_{1}\right)+\ldots+g_{k}\left(y_{m}\right) \equiv A\left(\bmod 2^{\gamma-\lambda}\right) .
$$

Note that $g_{k}(y)$ is an integral-valued polynomial. Also, $g_{k}(0)=0$ and $g_{k}(1) \not \equiv 0(\bmod 2)$, so that $g_{k}(y) \bmod 2$ is not constant. Thus, when $m=$ $2^{\gamma-\lambda}-1$ the congruence (4.6) is soluble for any $A$. Then, by (4.3) and (4.4) we have (cf. [7, Lemma 2.3])

$$
\Gamma\left(f_{k}, 2^{\gamma}\right) \leq\left(2^{\lambda}-1\right)+\left(2^{\gamma-\lambda}-1\right)=2^{\lambda}+2^{\gamma-\lambda}-2 .
$$


On the other hand, by (1.1), (4.5) and Taylor's expansion we see that the coefficient of $y^{k}$ in $g_{k}(y)$ is $a_{k} \cdot 2^{k-\lambda} / k$ ! . Then, writing $g_{k}(y)=\sum_{i=1}^{k} a_{i}^{\prime} F_{i}(y)$, we have $a_{k}^{\prime}=2^{k-\lambda} a_{k}$. We define $\mu$ by $2^{\mu} \| a_{k}$. By (2.1) and Lemma 2.2, $2^{\theta} \mid 2^{t} a_{k}$, and so $\theta \leq t+\mu$. Thus $a_{k}^{\prime}$ is divisible by 2 to the power $k-\lambda+\theta-t$, which is greater than or equal to $\gamma-\lambda$ by (2.2) and (2.3) (for $p=2$ ). Thus $g_{k}(y) \bmod 2^{\gamma-\lambda}$ is a polynomial of degree at most $k-1$. Then, by the induction hypothesis and the second assertion of Theorem 3(i), we see that when $m=2^{k-1}$ the congruence (4.6) is soluble. Hence

$$
\Gamma\left(f_{k}, 2^{\gamma}\right) \leq\left(2^{\lambda}-1\right)+2^{k-1} .
$$

Now (4.2) can be proved easily. Recall $\lambda \leq k-1$. If $\lambda \geq \delta+2$, then the function $2^{\lambda}+2^{\gamma-\lambda}$ of $\lambda$ has a maximum value at $\lambda=\delta+2$ or $\lambda=k-1$. It follows from (4.7), (2.2) and (2.4) (for $p=2$ ) that

$$
\Gamma\left(f_{k}, 2^{\gamma}\right) \leq 2^{k-1}+2^{\delta+2}-2 \leq 2^{k-1}+4(k-1)-2,
$$

as required. If $\lambda<\delta+2$, then (4.8) gives the result at once.

5. Proof of Theorem 4. We note that the case $p>k$ of Theorem 4 follows readily from Hua [3, Lemma 2.3]. Thus, to prove Theorem 4 it suffices to consider the cases when $3 \leq p \leq k$. We proceed by induction on $k \geq 5$. When $k=5$ the result has been proved in Yu [7, Section 6]. Suppose that the assertion of Theorem 4 is true for polynomials of degree $k-1(k \geq 6)$. We then prove

$$
\Gamma\left(f_{k}, p^{\gamma}\right) \leq 2^{k-1}+4(k-1)-1 \quad \text { for } 3 \leq p \leq k,
$$

and hence complete the proof. Since the argument of (5.1) is the same as that used in Section 4, we only give a brief sketch.

For $3 \leq p \leq k$, define $\lambda$ to be the greatest integer such that

$$
f_{k}(x+p)-f_{k}(x) \equiv 0\left(\bmod p^{\lambda}\right) \quad \text { for any } x .
$$

By Vandermonde's identity, we have

$$
f_{k}(x+p)-f_{k}(x)=\sum_{i=0}^{k-1} F_{i}(x) \sum_{j=1}^{k-i} a_{i+j}\left(\begin{array}{l}
p \\
j
\end{array}\right) .
$$

From this it can be proved that

$$
\lambda \leq\left[\frac{k-1}{p-1}\right]+1
$$

When $\gamma \leq \lambda$ the result is trivial. We thus assume that $\gamma>\lambda$. In analogy to (4.7) and (4.8) we have

$$
\Gamma\left(f_{k}, p^{\gamma}\right) \leq p^{\lambda}+p^{\gamma-\lambda}-2
$$


and (by the induction hypothesis, and using Hua's result mentioned above if $p=k$ )

$$
\Gamma\left(f_{k}, p^{\gamma}\right) \leq\left(p^{\lambda}-1\right)+\left(2^{k-2}+4(k-2)\right) .
$$

If $\lambda \geq \delta+1$, then the function $p^{\lambda}+p^{\gamma-\lambda}$ of $\lambda$ has a maximum value at $\lambda=\delta+1$ or $\lambda=\left[\frac{k-1}{p-1}\right]+1$ (cf. (5.2)). Then, by (5.3), (2.2) and (2.4) (for $p \geq 3$ ), it is easily verified that (5.1) holds for $6 \leq k \leq 10$ and

$$
\Gamma\left(f_{k}, p^{\gamma}\right)<p^{\left[\frac{k-1}{p-1}\right]+1}+p^{\delta+1} \leq p^{\frac{k-1}{p-1}+1}+k(k-1)<2^{k-1}+4(k-1)-1
$$

for $k \geq 11$. If $\lambda<\delta+1$, then (5.1) follows readily from (5.4).

Acknowledgements. The author is grateful to Professor M. G. Lu for suggesting this problem and for his encouragement.

\section{References}

[1] H. Gupta, Selected Topics in Number Theory, Abacus Press, 1980.

[2] L. K. Hua, On a generalized Waring problem, Proc. London Math. Soc. (2) 43 (1937), 161-182.

[3] -, On a generalized Waring problem, II, J. Chinese Math. Soc. 2 (1940), 175-191; see also L. K. Hua, Selected Papers, H. Halberstam (ed.), Springer, 1983, 61-73.

[4] - Additive Theory of Prime Numbers, Providence, R.I., 1965.

[5] L. Lovász, Combinatorial Problems and Exercises, North-Holland, Amsterdam, 1979.

[6] T. D. Wooley, On exponential sums over smooth numbers, J. Reine Angew. Math. 488 (1997), 79-140.

[7] H. B. Yu, On Waring's problem with polynomial summands, Acta Arith. 76 (1996), 131-144.

[8] —, On Waring's problem with quartic polynomial summands, ibid. 80 (1997), 77-82.

Department of Mathematics

University of Science and Technology of China

Hefei, Anhui 230026

The People's Republic of China

E-mail: yuhb@math.ustc.edu.cn 